\title{
A Measurement Method Research for the Ranging and Guiding System Based on the Rotating Magnetic Field
}

\author{
Xianfeng Gong \\ School of Mechanical Engineering \\ University of Science \& Technology Beijing, \\ Beijing, China \\ xianfenggong@126.com \\ Weibo Zhang \\ School of Mechanical Engineering \\ University of Science \& Technology Beijing, \\ Beijing, China \\ zwb601906695@163.com
}

\begin{abstract}
Objective: There exist some problems after analysising of the method existed. The writer present a new algorithm of the system of RMRS.

Methods: Analysis the signals in the three-axial fluxgate again, thereafter reconstruct the signal model. Based on the spectral modification and Lagrange Interpolation, the writer get the phase information between signals. Finally, what present to us are two equations which contain the unkown quantities, which can calculate the relative direction between the two horizontal wells. Combined with the RMRS system, the algorithm can provide parameters to traditional logging system, thus we can get the relative location between the double horizontal well. Results: We program the algorithm and verify it by lots of locations. The experimental result is totally meeting the precision. Conclusion: This method can measure the relative position at any point within the signal's effective range, greatly enhanced the convenience of RMRS's operation.
\end{abstract}

Keywords SAGD; twin horizontal; magnetic dipole; phase difference; spectral modification;

\author{
Yong Zhu \\ School of Mechanical Engineering \\ University of Science \& Technology Beijing \\ Beijing, China \\ 501719851@qq.com
}

\section{INTRODUCTION}

Oil is the blood of industry, it is of great significance to enhanced Oil Recovery. Nowadays, as the resources of crude oil which have good liquidity are drying up, people pay more attention to the heavy oil or super heavy oil, which has the characteristics of big density and poor liquidity. The International cutting-edge technology SAGD is developed in 80 's, which is particularly suitable for heavy crude oil or natural asphalt. In order to guarantee the successful application of this technology, it is necessary to keep two wells in parellel conditon and the offset error between them within $+-1.0 \mathrm{~m}$. Meanwhile, the relative azimuth angle error should control in $+-5^{\circ}$. In order to meet this requirement, there are mainly two guidance system nowadays: rotating magnetic ranging system (RMRS) and magnetic orientation tools (MGT), which have been widely applied[1-3] .

Kuckes and others[4-5] proposed a algorithm at literature which is widely used based on the system of RMRS. However, this algorithm is just effective when the magnetic sub directly below the test tube. It's effective measure range is very 
limited. In practice, it relays on manual work to transport the test tube, hard in operating. In allusion to the problems mentioned above, the writer proposes another algorithm after analyzing the signal distributed by magnetic sub carefully, which is effective to this problem.

The rotating magnetic field ranging system is mainly composed of the magnetic field source, test tube and a data analysis software. The magnetic sub is the magnetic source, generating the low frequency alternating magnetic field. The test tube measure the magnetic field signal which will final transfer to the computer for analysis. The date analysis software can make use of the signal and calculate the distance and relative direction.

Just as shown in Fig 1. The test tube downhole is the core of the rotating magnetic field ranging system, which based on the Microcontroller. The signals from flux-gate sensors, acceleration sensors, temperature sensors and well depth measurement system will be disposed and quantified. Through some certain communication protocol, the interface box will receive these finals and pass it to computer. Thus the relative direction will present to us after a certain arithmetic.

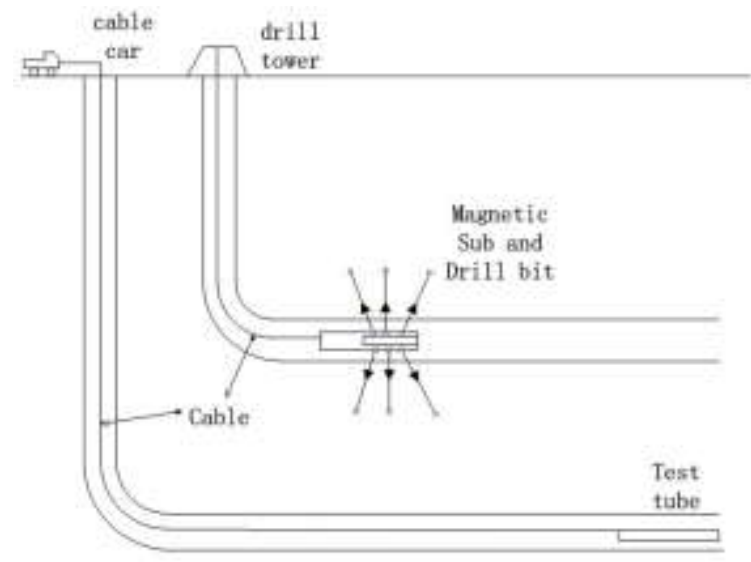

Figure 1. The system of the rotating magnet for distance and direction

\section{THE SIGNAL MODEL OF MAGNETIC SUB}

In actual production, we consider the rotating magnetic sub as rotating magnetic dipole, because it is often that the size of the magnetic sub is relatively small than the horizontal distance between the magnetic sub and test tube. For the convenience of calculation, we establish a coordinate system as follows: taking the direction of the steam injection well's bore as the axis of $z$, the direction from steam injection well to production well as the axis of $r$, the axis of $q$ is in orthogonal with two axises above. These unit vectors are perpendicular to each other.

Diao Binbin and others[6] have derived out the component of the magnetic induction intensity which produced by magnetic sub, may as follows:

$$
\begin{aligned}
& B_{r}=\frac{M\left(2 r^{2}-z^{2}\right) \cos A_{m r}}{4 \pi\left(r^{2}+z^{2}\right)^{\frac{5}{2}}} \\
& B_{q}=\frac{M \sin A_{m r}}{4 \pi\left(r^{2}+z^{2}\right)^{\frac{3}{2}}}
\end{aligned}
$$

$$
B_{z}=\frac{3 M r z \cos A_{m r}}{4 \pi\left(r^{2}+z^{2}\right)^{\frac{5}{2}}}
$$

$$
\begin{aligned}
& \text { Amr }=\omega t-A t r, \omega \text { being the } \\
& \text { motor's angular velocity, which driving the } \\
& \text { magnetic sub. When t equals } 0 \text {, the intersection } \\
& \text { angle between magnetic torque and } r \text { is Atr. At } \\
& \text { the moment, Amr= } \omega t
\end{aligned}
$$


III. THE ALGORITHM OF RANGING GUIDANCE FOR THE ROTATING MAGNETIC FIELD RANGING GUIDING SYSTEM

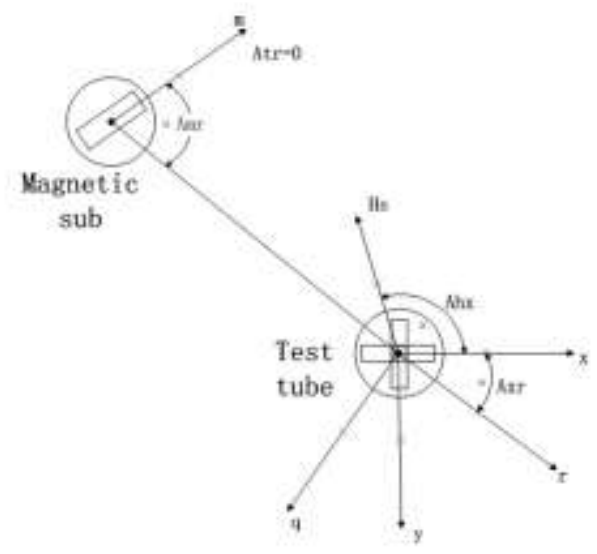

Figure 2. The relative position of twin horizontal wells

A. The signal in triaxial flux-gate sensor

Just as shown in figure 2 , the unit vector $\overrightarrow{\mathrm{x}}, \overrightarrow{\mathrm{y}}$, and $\overrightarrow{\mathrm{z}}$ represent the direction vector $\mathrm{x}$, $\mathrm{y}$ and $\mathrm{z}$ which are the vector of triaxial flux-gate sensor. Hs being the drilled well's direction of high side. Ahr being the angle from Hs to unit vector $\vec{r}$. The tool angle of gravity Ahx called tool angle of high side as well, which defined as the angle rotating clockwise from high side to vector $\mathrm{x}$ when overlooking the well. The Axr being the angle from unit vector $\vec{x}$ to unit vector $\vec{r}$. The relative direction of double horizontal well can be determined by Ahr, which equals Ahx plus Axr.

According to Diaobinbin's article:

$$
\begin{aligned}
& B_{x}=B_{r} \cos A_{x r}-B_{q} \sin A_{x r} \\
& B_{y}=B_{r} \sin A_{x r}+B_{q} \cos A_{x r}
\end{aligned}
$$

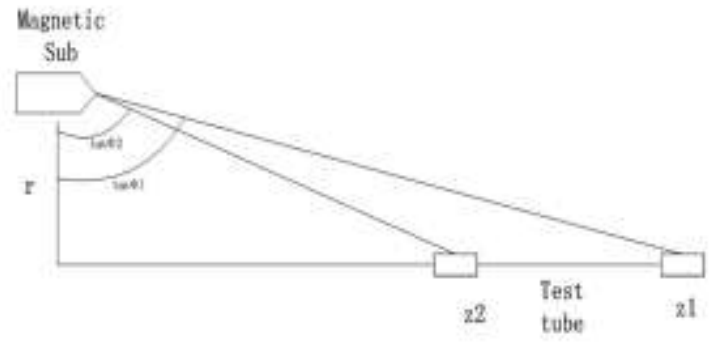

Figure 3. the calculation model of magnetic sub and

$$
\text { test tube }
$$

Look from the axis of q's normal direction, shown as Fig 3, set

$$
\mathrm{z}=\mathrm{rtan} \theta
$$

$\mathrm{z}$ being the distance from magnetic sub to test tube along the direction $\vec{z} ; \mathrm{z} 1$ being the distance at point $1 ; \mathrm{z} 2$ being the distance at point $2 ; \mathrm{r}$ being the offset between two wells; $\tan \theta 1$ being the value of tangent between magnetic sub and test tube at point $1 ; \tan \theta 2$ being the value of tangent between magnetic sub and test tube at point 2.

Take point 1 as example. Put the equation of (1), (2), (3), (6) into (4), (5). The magnetic induction intensity at point 1 can be concluded as:

$$
\mathrm{Bx}=\frac{M}{4 \pi r^{3}\left(1+\tan ^{2} \theta 1\right)^{\frac{5}{2}}}\left[\left(2-\tan ^{2} \theta 1\right) \cos A m r\right.
$$$$
\left.\cos A x r 1-\left(1+\tan ^{2} \theta 1\right) \sin A m r \sin A x r 1\right]
$$

$$
\begin{aligned}
& \mathrm{By}=\frac{M}{4 \pi r^{3}\left(1+\tan ^{2} \theta 1\right)^{\frac{5}{2}}}\left[\left(2-\tan ^{2} \theta 1\right) \cos A m r\right. \\
& \left.\sin A x r 1-\left(1+\tan ^{2} \theta 1\right) \sin A m r \cos A x r 1\right]
\end{aligned}
$$

$$
\mathrm{B}_{z}=\frac{3 M \tan \theta 1 \cos A m r}{4 \pi r^{3}\left(1+\tan ^{2} \theta 1\right)^{\frac{5}{2}}}
$$

According to the triangle formula:

$$
\left\{\begin{array}{l}
\cos (A+B)=\cos (A) \cos (B)-\sin (A) \sin (B) \\
\sin (A+B)=\sin (A) \cos (B)+\cos (A) \sin (B)
\end{array}\right.
$$

For further derivation: 


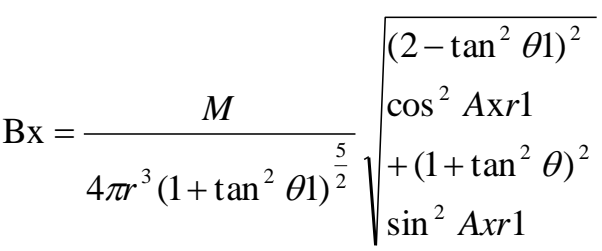

$\cos (A m r-\mathrm{p} a)$

(11)

We work out the phase difference by FFT. In practice, it has certain effect in interference and noise. In order to enhance precision, we process a spectrum modification at the same time. [7-8]

Take the signale $\mathrm{Bx}$ as example. In this article, we adapt hanning-window as the truncation window, process discrete Fourier of Bx. After spectral modificatin, finally we can get phase value of signal $B x$. Seeing from the equation (13), the phase of signal $\mathrm{Bz}$ always been zero. The phase difference $\mathrm{Pa}$ between signal $\mathrm{Bx}$ $\cos (A m r-\mathrm{pb})$

$$
\mathrm{B}_{z}=\frac{3 M \tan \theta 1 \cos A m r}{4 \pi r^{3}\left(1+\tan ^{2} \theta 1\right)^{\frac{5}{2}}}
$$

Among them:

$$
\operatorname{cospa}=\frac{\left(2-\tan ^{2} \theta 1\right) \cos A x r 1}{\sqrt{\frac{\left(2-\tan ^{2} \theta 1\right)^{2} \cos ^{2} A x r 1+\left(1+\tan ^{2} \theta 1\right)^{2}}{\sin ^{2} A x r 1}}}
$$
and $\mathrm{Bz}$ can be deducted. By the same token, we can get the phase difference $\mathrm{Pb}$ between signal $\mathrm{By}$ and $\mathrm{Bz}$.

\section{B. The algorithm to working out the relative position}

Set $\mathrm{x}=\tan ^{2} \theta 1, y=\cos ^{2} A x r 1$, substitute them into equation (14) and (16).

$$
\begin{aligned}
& \frac{(2-x)^{2} y}{(2-x)^{2} y+(1+x)^{2}(1-y)}=\cos ^{2} P a=a \\
& \frac{(2-x)^{2}(1-y)}{(2-x)^{2}(1-y)+(1+x)^{2} y}=\cos ^{2} P b=b
\end{aligned}
$$

$$
\begin{aligned}
& \sin p a=\frac{-\left(1+\tan ^{2} \theta 1\right) \sin A x r 1}{\sqrt{\begin{array}{l}
\left(2-\tan ^{2} \theta 1\right)^{2} \cos ^{2} A x r 1+\left(1+\tan ^{2} \theta 1\right)^{2} \\
\sin ^{2} A x r 1
\end{array}}} \text { It can be concluded that: } \\
& y=\frac{a(1+x)^{2}}{x^{2}+(6 a-4) x+4-3 a}
\end{aligned}
$$

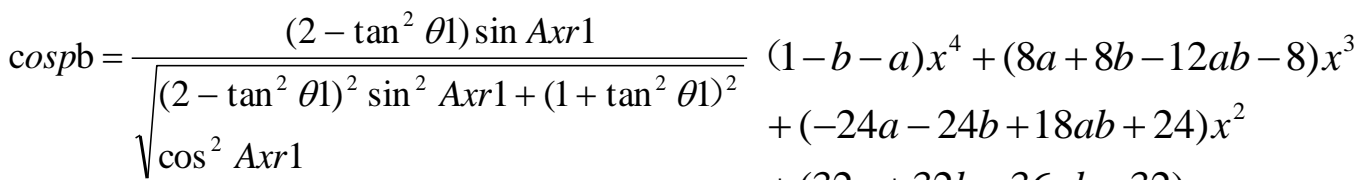

$$
\begin{aligned}
& +(32 a+32 b-36 a b-32) x \\
& +(-16 a-16 b+15 a b+16)=0
\end{aligned}
$$

$$
\sin p \mathrm{~b}=\frac{\left(1+\tan ^{2} \theta 1\right) \cos A x r 1}{\sqrt{\begin{array}{l}
\left(2-\tan ^{2} \theta 1\right)^{2} \sin ^{2} A x r 1+\left(1+\tan ^{2} \theta 1\right)^{2} \\
\cos ^{2} A x r 1
\end{array}}}
$$

$\mathrm{Pa}$ being the phase difference between $\mathrm{Bx}$ and $\mathrm{Bz}, \mathrm{Pb}$ being the phase difference between $\mathrm{By}$ and $\mathrm{Bz}$. 
Axr's interval relative to the value and direction of $\operatorname{cospa}$, $\sin \mathrm{Pa}, \cos \mathrm{Pb}, \sin \mathrm{Pb}$ and $\tan \theta$.

Just as shown in fig 3, we can analysis and process signal at point 2 just as point 1 . Calculating the value of $\tan \theta 2$ and $\cos A x r 2$. The relative angle is $\Delta \tan \theta=\tan \theta 1-\tan \theta 2$. The relative depth z1-z2 can be measured by the system of measuring well's depth, which is a part of MWD. Thus the value of $r$ can be worked out.

$$
\begin{aligned}
& \tan \theta 1-\tan \theta 2=\frac{z 1}{r}-\frac{z 2}{r} \\
& \mathrm{r}=\frac{z 1-z 2}{\tan \theta 1-\tan \theta 2}
\end{aligned}
$$

The system had the special circuit for triaxial acceleration sensor, which can detect the value of Ahx, thus the value of Ahr. Combined with the

\begin{tabular}{|c|c|c|c|c|c|c|}
\hline & $\begin{array}{r}0 . \\
8391 \\
( \\
\left.40^{\circ}\right)\end{array}$ & $\begin{array}{r}0 . \\
5774 \\
( \\
\left.30^{\circ}\right)\end{array}$ & $\begin{array}{r}1 . \\
1918 \\
( \\
\left.50^{\circ}\right)\end{array}$ & $\begin{array}{r}0 . \\
8391 \\
( \\
\left.40^{\circ}\right)\end{array}$ & $\begin{array}{r}1 . \\
7312 \\
( \\
\left.60^{\circ}\right)\end{array}$ & $\begin{array}{r}1 . \\
1918 \\
( \\
\left.50^{\circ}\right)\end{array}$ \\
\hline $0^{\circ}$ & $\begin{array}{r}\tan \\
0.8325 \\
\tan \\
0.5610 \\
\mathrm{r} \\
4.8210 \\
\mathrm{Ax} \\
19.668\end{array}$ & & $\begin{array}{r}\tan \\
1.1909 \\
\tan \\
0.8325 \\
\mathrm{r} \\
4.9207 \\
\mathrm{Ax} \\
19.90\end{array}$ & & $\begin{array}{r}\tan \\
1.730 \\
\tan \\
1.1909 \\
\mathrm{r} \\
5.0066 \\
\mathrm{Ax} \\
20.099\end{array}$ & \\
\hline $0^{\circ}$ & $\begin{array}{r}\tan \\
0.8376 \\
\tan \\
0.5737 \\
\mathrm{r}\end{array}$ & & $\begin{array}{r}\tan \\
1.1916 \\
\tan \\
0.8376 \\
r\end{array}$ & & $\begin{array}{r}\tan \\
1.731 \\
\tan \\
1.1916 \\
\mathrm{r}\end{array}$ & \\
\hline
\end{tabular}

TABLE 1. THE RESULTS OF DIFFERENT POSTURE

\begin{tabular}{|c|c|c|c|}
\hline & 4.9579 & 4.9822 & 5.0010 \\
$\mathrm{Axr}$ & $\mathrm{Axr}$ & $\mathrm{Axr}$ \\
39.7001 & 39.9077 & 40.0975 \\
\hline $0^{\circ}$ & $\tan \theta 1$ & $\tan \theta 1$ & $\tan \theta 1$ \\
& 0.8409 & 1.1920 & 1.7327 \\
& $\tan \theta 2$ & $\tan \theta 2$ & $\tan \theta 2$ \\
0.5815 & 0.8409 & 1.1920 \\
& $\mathrm{r}$ & $\mathrm{r}$ & $\mathrm{r}$ \\
5.0455 & 5.0217 & 4.9962 \\
& Axr & Axr & Axr \\
& 59.7332 & 59.9151 & 60.0938 \\
& & \\
\hline
\end{tabular}

results of traditional method to calculating the wellbore trajectory. Finally we can get the test tube's relative space position[9-10].

\section{THE VALIDATION AND ANALYSIS BY EXAMPLE}

The signal of $\mathrm{Bx}, \mathrm{By}, \mathrm{Bz}$, which received by three-axial fluxgate are standard cosine signals. Thereby we can add a random noise. Fixxing the value of $M 、 r$ and $\omega$, setting the vale of $\tan \theta$ and Axr when modeling as well. After deducing and calculating carefully, verifying the effective of the algorithm by compare the vlue of $r, \tan \theta$ and $A x r$ with preestablished value. The result just as Table 1 shown.

In this example, we set the value of $\mathrm{M}$ being 10, $\mathrm{r}$ being $5, \omega$ being $150 \mathrm{r} / \mathrm{min}$. The value of horizontal axis is $\tan \theta 1$ and $\operatorname{an} \theta 2$, and the verticle axis is Axr.

The triaxial signals at point $\mathrm{z} 1$ and $\mathrm{z} 2$ are shown in figure 4 and figure 5 : 

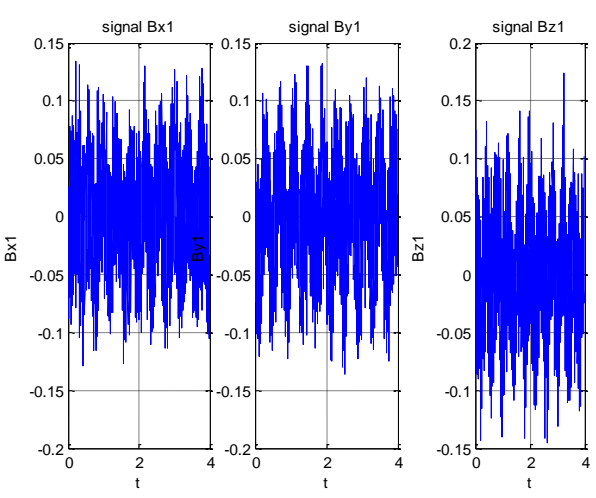

Figure 4. signals detected by test tube at point $\mathrm{z} 1$

Writing a program as present above and calculating orientation under different posture. It can verify the validity of this algorithem by table 1 , and the precision totally meet the engineering application.

\section{CONLUSION}

This article utilize the relationship of phase among the three-axial fluxgate to deduce the relative oriention. It have deduced the physic model of rotating magnetic dipole, analysised the signal received by three-axial fluxgate. Thereafter, the equations for relative oriention present to us. If this algorithm applied, it has the characteristics of without detecting the value of magnetic moment, working out the relative oriention at arbitrarily space position when signal is effective, easy operation and good precision. The example verify the algorithm's availability, high application value in engineering as well.

\section{REFERENCE}

[1] Heavy oils: reservoir characterization and production monitoring $[\mathrm{M}]$. Society of Exploration Geophysicists, 2010.

[2] Hosseininejad Mohebati M, Maini B, Harding T. Numerical-Simulation Investigation of the Effect of Heavy-Oil Viscosity on the Performance of Hydrocarbon Additives in SAGD[J]. SPE Reservoir Evaluation \& Engineering, 2012, 15(2): 165-181.

[3] Sharma J, Gates I. Convection at the Edge of a Steam-Assisted-Gravity-Drainage Steam Chamber[J]. SPE Journal, 2011, 16(3): 503-512.

[4] Kuckes, Arthur F. "Rotating magnet for distance and direction measurements from a first borehole to a second borehole." U.S. Patent No. 5,589,775. 31 Dec. 1996.

[5] Kuckes, Arthur F. "Method for near field electromagnetic proximity determination for guidance
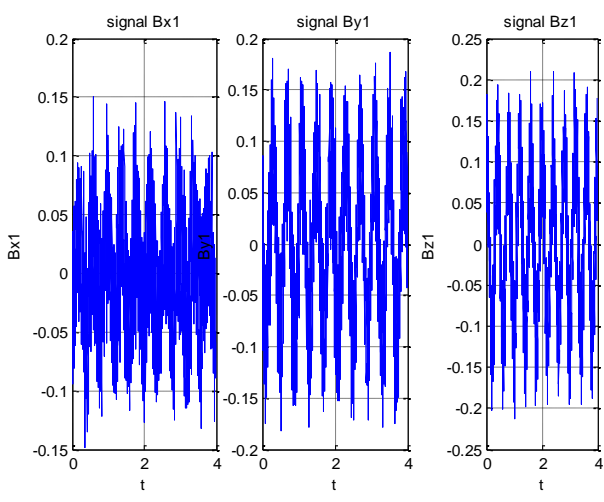

Figure 5. signals detected by test tube at point z2 of a borehole drill." U.S. Patent No. 5,923,170. 13 Jul. 1999.

[6] Diao Bin-bin , Gao De-li, Wu Zhi-yong ,et al. The oriented algorithm of magnetic distance beween two horizontal wells $[\mathrm{J}]$. Journal of China university of petroleum (Natural Science ) ,2011,35(6):71-75.

[7] Xie Ming, Ding Kang. A new rectifying technique of discrete spectrum analysis [J]. Journal of Chongqing university (Natural Science Edition) ,1995,(3):47-54.

[8] Zhibo L, Linsong C, Youjun J, et al. Production features of steam and gas push: Comparative analysis with steam assisted gravity drainage[J]. Petroleum Exploration and Development, 2011, 38(1): 79-83.

[9] Cokar M, Gates I, Kallos M. A New Thermogeomechanical Theory for Gravity Drainage in Steam-Assisted Gravity Drainage[J]. SPE Journal, 2013, 18(4): 736-742.

[10] Kapadia P R, Wang J, Kallos M S, et al. Thermal-reactive reservoir engineering model predicts Hydrogen Sulfide GenerationinSteam assisted gravity drainage[J]. Journal of Petroleum Science and Engineering, 2012.

[11] G. Eason, B. Noble, and I. N. Sneddon, "On certain integrals of Lipschitz-Hankel type involving products of Bessel functions," Phil. Trans. Roy. Soc. London, vol. A247, pp. 529-551, April 1955. (references) 